\title{
A138 APOE-DEFICIENT MICE ARE RESISTANT TO THE DEVELOPMENT OF COLLAGEN-INDUCED ARTHRITIS
}

D L Asquith, A M Miller, A J Hueber, F Y Liew, N Sattar, I B McInnes University of Glasgow, Glasgow, UK

10.1136/ard.2010.1296430

A study was undertaken to determine whether elevated serum lipid levels induced by high fat diet feeding can affect the inflammatory processes in collagen-induced arthritis (CIA) models in C57BL/6 wild-type (WT) and C57BL/6 ApoE-/mice. CIA was induced in male B6 WT or ApoE-/- mice, fed either normal chow diet or high fat diet, at 12 weeks of age using Type II chicken collagen, Freund's complete adjuvant and on occasion LPS boost. Autoantibody and cytokine expression were measured by ELISA and multiplex respectively. Whereas B6 WT mice developed severe articular inflammation after collagen immunisation, ApoE-/- mice developed no clinical or histological evidence of disease whether receiving a high fat diet or normal chow diet. This lack of arthritis was not due 
to an inadequate production of serum IgG2a collagen antibodies, as levels were similar to those in arthritic B6 WT control mice. Critically, atherosclerosis development in ApoE-/- mice was not affected by the CIA protocol. In conclusion, ApoE-/mice appear resistant to the development of collagen-induced arthritis. Intriguingly, induction of host autoimmunity in the absence of articular inflammation had no effect on atherosclerosis progression, suggesting that the articular inflammatory load may be a critical risk factor in vascular pathology. 